\title{
Relationships between initial unloading slope, contact depth, and mechanical properties for conical indentation in linear viscoelastic solids
}

\author{
Yang-Tse Cheng ${ }^{\text {a) }}$ \\ Materials and Processes Laboratory, General Motors Research and Development Center, \\ Warren, Michigan 48090 \\ Che-Min Cheng \\ Institute of Mechanics, Chinese Academy of Sciences, Beijing 100080, People's Republic of China
}

(Received 18 December 2004; accepted 26 January 2005)

\begin{abstract}
Using analytical and finite element modeling, we studied conical indentation in linear viscoelastic solids with either displacement or load as the independent variable. We examine the relationships between initial unloading slope, contact depth, and viscoelastic properties for various loading conditions such as constant displacement rate, constant loading rate, and constant indentation strain rate. We then discuss whether the Oliver-Pharr method for determining contact depth, originally proposed for indentation in elastic and elastic-plastic solids, is applicable to indentation in viscoelastic solids. We conclude with a few comments about two commonly used experimental procedures for indentation measurements in viscoelastic solids: the "hold-at-peak-load" technique and the constant indentation strain-rate method.
\end{abstract}

\section{INTRODUCTION}

Instrumented indentation is playing an increasing role in the study of small-scale mechanical behavior of "soft" matters, such as polymers, composites, biomaterials, and food products. Many of these materials exhibit viscoelastic behavior, especially at elevated temperatures. Modeling of indentation into viscoelastic solids thus forms the basis for analyzing indentation experiments in these materials. Theoretical studies of contacting linear viscoelastic bodies became active since the mid 1950s by the work of Lee, ${ }^{1}$ Radok, ${ }^{2}$ Lee and Radok, ${ }^{3}$ Hunter, ${ }^{4}$ Gramham, ${ }^{5,6}$ Yang, ${ }^{7}$ and Ting. ${ }^{8,9}$ In recent years, a number of authors have extended the early work to the analysis of indentation measurements in viscoelastic solids. ${ }^{10-15}$ In this paper, we examine, through analytical and finite element modeling, the relationship between initial unloading slope, contact depth, and viscoelastic properties. We then discuss whether the commonly used Oliver-Pharr method $^{16,17}$ is applicable to indentation in viscoelastic

\footnotetext{
a) Address all correspondence to this author.

e-mail: yang.t.cheng@gm.com

This author was an editor of this journal during the review and decision stage. For the $J M R$ policy on review and publication of manuscripts authored by editors, please refer to http:// www.mrs.org/publications/jmr/policy.html.

DOI: $10.1557 / J M R .2005 .0141$
}

solids. We conclude with a critique of two commonly used experimental procedures, the "hold-at-peak-load" technique and the constant indentation strain-rate method, for indentation measurements in viscoelastic solids.

\section{ANALYTICAL RESULTS}

\section{A. Conical indentation in linear viscoelastic solids}

We consider a rigid, smooth, and frictionless conical indenter with half-angle $\theta$ indenting a viscoelastic solid that can be described by the following constitutive relationships ${ }^{18,19}$ between deviatoric stress and strain, $s_{\mathrm{ij}}$ and $d_{\mathrm{ij}}$, and between dilatational stress and strain, $\sigma_{\mathrm{ii}}$ and $\epsilon_{\mathrm{ii}}$,

$$
\begin{aligned}
s_{\mathrm{ij}}(t) & =2 \int_{0}^{t} \mathrm{G}(t-\tau) \frac{\partial \mathrm{d}_{\mathrm{ij}}(\tau)}{\partial \tau} \mathrm{d} \tau \\
\sigma_{\mathrm{ii}}(t) & =3 \int_{0}^{t} K(t-\tau) \frac{\partial \epsilon_{\mathrm{ii}}(\tau)}{\partial \tau} \mathrm{d} \tau
\end{aligned}
$$

where $G(t)$ is the relaxation modulus in shear and $K(t)$ is the relaxation modulus in dilatation. The time dependent Young's modulus and Poisson's ratio are then given by $E(t)=[9 K(t) G(t)] /[3 K(t)+G(t)]$ and $\nu(t)=[E(t) /$ $2 G(t)]-1$, respectively. 
Alternatively, the stress-strain relations can be written as $^{18,19}$

$$
\begin{aligned}
2 \mathrm{~d}_{\mathrm{ij}}(t) & =\int_{0}^{t} J_{1}(t-\tau) \frac{\partial s_{\mathrm{ij}}(\tau)}{\partial \tau} \mathrm{d} \tau \\
3 \epsilon_{\mathrm{ii}}(t) & =\int_{0}^{t} J_{2}(t-\tau) \frac{\partial \sigma_{\mathrm{ii}}(\tau)}{\partial \tau} \mathrm{d} \tau,
\end{aligned}
$$

where $J_{1}(t)$ is the shear compliance and $J_{2}(t)$ is the volumetric compliance. Obviously, Eqs. (1) and (2) are not independent of each other. In fact, the relationships between the relaxation and creep functions, $G(t)$ and $J_{1}(t)$, as well as $K(t)$ and $J_{2}(t)$, have simple forms after transforming them using Laplace transformation, ${ }^{18,19}$

$$
\begin{aligned}
& \int_{0}^{t} J_{1}(t-\tau) G(\tau) \mathrm{d} \tau=\int_{0}^{t} J_{1}(\tau) G(t-\tau) \mathrm{d} \tau=t \\
& \int_{0}^{t} J_{2}(t-\tau) K(\tau) \mathrm{d} \tau=\int_{0}^{t} J_{2}(\tau) K(t-\tau) \mathrm{d} \tau=t .
\end{aligned}
$$

As a consequence, we have,

$$
\begin{aligned}
& J_{1}(0) G(0)=J_{1}(\infty) G(\infty)=1 \\
& J_{2}(0) K(0)=J_{2}(\infty) K(\infty)=1 .
\end{aligned}
$$

In the following, we assume that Poisson's ratio is time independent. Consequently, $G(t)$ and $v$ are sufficient to describe the linear viscoelastic behavior.

\section{B. Relationships between load, displacement, and contact depth}

With displacement as the independent variable, the relationship between force $F(t)$ and displacement $h(t)$ is then given by ${ }^{1-9,20}$

$$
F(t)=\frac{8 \tan \theta}{\pi(1-\nu)} \int_{0}^{t} G(t-\tau) h(\tau) \frac{\mathrm{d} h(\tau)}{\mathrm{d} \tau} \mathrm{d} \tau .
$$

With force as the independent variable, the relationship between displacement $h(t)$ and force $F(t)$ is given by $^{1-9}$

$$
h^{2}(t)=\frac{\pi(1-\nu)}{4 \tan \theta} \int_{0}^{t} J_{1}(t-\tau) \frac{\mathrm{d} F(\tau)}{\mathrm{d} \tau} \mathrm{d} \tau .
$$

The load-displacement relationship can therefore be obtained if the viscoelastic properties of materials, $G(t)$ or $J_{1}(t)$, and $v$, are known using the respective Eqs. (5) or (6). Conversely, the viscoelastic properties may be obtained from measured $F(t)$ versus $h(t)$ relations by solving integral Eqs. (5) or (6). ${ }^{10-15}$ Equations (5) and (6) reduce to the well-known equation for conical indentation into purely elastic solids ${ }^{21}$

$$
F=\frac{4 G \tan \theta}{\pi(1-v)} h^{2},
$$

where $G=1 / J_{1}$ and $v$ are the time-independent shear modulus and Poisson's ratio, respectively.

Equations (5) and (6) are special cases of more general expressions derived by Graham ${ }^{5}$ and Ting. ${ }^{8}$ They showed that Eqs. (5) and (6) are valid when the contact area is a monotonically increasing function of time. Under the same condition, the ratio of contact depth to indenter displacement is the same as that in the purely elastic case $\mathrm{cos}^{5,8}$

$$
\frac{h_{\mathrm{c}}}{h}=\frac{2}{\pi} \text {. }
$$

\section{Relationships between initial unloading slope, contact depth, and viscoelastic property}

The equations for unloading where the contact area decreases monotonically have also been derived, ${ }^{5,8}$ though they are more complicated. As a result, a number of authors have proposed methods for deducing $G(t)$ or $J_{1}(t)$ from indentation loading curves using Eqs. (5) or (6) without using the information contained in the indentation unloading curves. ${ }^{20}$ However, we have recently shown ${ }^{20}$ that Eqs. (5) and (8) can be used to evaluate the initial unloading slope of unloading curves when displacement is the independent variable. Suppose unloading takes place at $t=t_{\mathrm{m}}^{+}$with a constant unloading rate of $\mathrm{d} h / \mathrm{d} t \mid t_{\mathrm{m}}^{+}=-v_{\mathrm{h}}$, we have, using Eqs. (5) and (8) for 0 $\leqslant t_{\mathrm{m}} \leqslant t_{\mathrm{m}}+\Delta t$ and $\Delta t \rightarrow 0$,

$$
\begin{gathered}
\left.\frac{\mathrm{d} F}{\mathrm{~d} h}\right|_{h=h_{\mathrm{m}}}=\frac{4 \tan \theta}{1-v}\left[\mathrm{G}(0) h_{\mathrm{c}}\left(t_{\mathrm{m}}^{+}\right)-\left.\frac{2}{\pi \nu_{\mathrm{h}}} \int_{0}^{t_{\mathrm{m}}} \frac{\mathrm{d} G}{\mathrm{~d} \eta}\right|_{\eta=t_{\mathrm{m}}-\tau}\right. \\
\left.h(\tau) \frac{\mathrm{d} h(\tau)}{\mathrm{d} \tau} \mathrm{d} \tau\right] .
\end{gathered}
$$

The second term on the right-hand side of Eq. (9) is a function of loading history. When the second term is negligible compared to the first term, which can be achieved by using fast unloading (i.e., large $v_{\mathrm{h}}$ ), the relationship between unloading slope and contact area becomes identical to that for purely elastic contacts ${ }^{16,21}$

$\left.\frac{\mathrm{d} E}{\mathrm{~d} h}\right|_{h=h_{\mathrm{m}}}=\frac{4 G(0) \tan \theta}{1-v} h_{\mathrm{c}}=\frac{2}{\sqrt{\pi}} \frac{E(0)}{1-v^{2}} \sqrt{A}$,

where $A=\pi\left(h_{\mathrm{c}} \tan \theta\right)^{2}$ is the contact area at time $t_{\mathrm{m}}$, when unloading takes place from $h_{\mathrm{m}}$.

An equation similar to Eq. (9) can also be derived for 
situations where force is the independent variable. Suppose unloading takes place at $t=t_{\mathrm{m}}^{+}$with a constant unloading rate of $\mathrm{d} F / \mathrm{d} t \mid t_{\mathrm{m}}^{+}=-v_{F}$, we have, using Eq. (6) for $0 \leqslant t \leqslant t_{\mathrm{m}}+\Delta t$ and $\Delta t \rightarrow 0$,

$$
\begin{aligned}
\frac{h^{2}\left(t_{\mathrm{m}}+\Delta t\right)-h^{2}\left(t_{\mathrm{m}}\right)}{\Delta t} & \frac{\pi(1-v)}{4 \tan \theta \Delta t}\left[\int_{0}^{t_{\mathrm{m}}+\Delta t} J_{1}\left(t_{\mathrm{m}}+\Delta t-\tau\right) \frac{\mathrm{d} F(\tau)}{\mathrm{d} \tau} \mathrm{d} \tau\right. \\
& \left.-\int_{0}^{t_{\mathrm{m}}} J_{1}\left(t_{\mathrm{m}}-\tau\right) \frac{\mathrm{d} F(\tau)}{\mathrm{d} \tau} \mathrm{d} \tau\right] \\
= & \frac{\pi(1-v)}{4 \tan \theta \Delta t}\left[\int_{0}^{t_{\mathrm{m}}} J_{1}\left(t_{\mathrm{m}}-\tau\right) \frac{\mathrm{d} F(\tau)}{\mathrm{d} \tau} \mathrm{d} \tau\right. \\
+ & \left.\Delta t \int_{0}^{t_{\mathrm{m}}} \frac{\mathrm{d} J_{1}(\eta)}{\mathrm{d} \eta}\right|_{\eta=t_{\mathrm{m}}-\tau} \frac{\mathrm{d} F(\tau)}{\mathrm{d} \tau} \mathrm{d} \tau+J_{1}(0) \frac{\mathrm{d} F\left(t_{\mathrm{m}}^{+}\right)}{\mathrm{d} t} \Delta t \\
& \left.-\int_{0}^{t_{\mathrm{m}}} J_{1}\left(t_{\mathrm{m}}-\tau\right) \frac{\mathrm{d} F(\tau)}{\mathrm{d} \tau} \mathrm{d} \tau\right] \\
= & \frac{\pi(1-v)}{4 \tan \theta}\left[\left.\int_{0}^{t_{\mathrm{m}}} \frac{\mathrm{d} J_{1}(\eta)}{\mathrm{d} \eta}\right|_{\eta=t_{\mathrm{m}}-\tau} \frac{\mathrm{d} F(\tau)}{\mathrm{d} \tau} \mathrm{d} \tau\right. \\
& \left.J_{1}(0) v_{\mathrm{F}}\right] \cdot
\end{aligned}
$$

The initial unloading slope is then given by, using

$$
\frac{\mathrm{d} F}{\mathrm{~d} h}=\frac{\mathrm{d} F}{\mathrm{~d} t} / \frac{\mathrm{d} h}{\mathrm{~d} t}=-v_{\mathrm{F}} / \frac{\mathrm{d} h}{\mathrm{~d} t}=-2 h v_{\mathrm{F}} / \frac{\mathrm{d} h^{2}}{\mathrm{~d} t}
$$

and Eq. (8),

$$
\begin{gathered}
\left.\frac{\mathrm{d} F}{\mathrm{~d} h}\right|_{h=h_{\mathrm{m}}}=\frac{4 h_{\mathrm{c}} \tan \theta}{1-v} \frac{1}{J(0)-\left.\frac{1}{\nu_{\mathrm{F}}} \int_{0}^{t_{\mathrm{m}}} \frac{\mathrm{d} J_{1}(\eta)}{\mathrm{d} \eta}\right|_{\eta=t_{\mathrm{m}}-\tau} \frac{\mathrm{d} F(\tau)}{\mathrm{d} \tau} \mathrm{d} \tau} \\
v_{F} \rightarrow \infty \\
\approx \frac{4 h_{\mathrm{c}} \tan \theta}{1-v} \frac{1}{J_{1}(0)} .
\end{gathered}
$$

When the unloading rate $v_{\mathrm{F}}$ is much larger than the loading history dependent integral

$$
\int_{0}^{t_{\mathrm{m}}}\left[\mathrm{d} J_{1}(\eta) /\left.\mathrm{d} \eta\right|_{\eta^{\prime}=t_{\mathrm{m}^{-}}}\right][\mathrm{d} F(\tau) / \mathrm{d} \tau] \mathrm{d} \tau,
$$

the relationship between unloading slope and contact depth or area is again the same as that for purely elastic contacts [Eq. (10)].

Eqs. (9) and (12) suggest that the initial unloading slopes converge when the unloading rate, either in displacement or load control, is sufficiently fast. Once this limiting case is reached, Eq. (10) can be used to determine the "instantaneous" moduli, $G(0) /(1-v)$ or $E(0) /\left(1-v^{2}\right)$, provided that the contact depth, $h_{\mathrm{c}}$ or area $A$ is known as a function of $h_{\mathrm{m}}=h\left(t_{\mathrm{m}}\right)$. The latter condition is provided by Eq. (8). An interesting, though not necessarily surprising, implication of Eqs. (9) and (12) is that the details of loading history, i.e., $h(t)$ or $F(t)$ in the respective displacement- or load-controlled measurements, are unimportant as long as the unloading rate is sufficiently fast. A sufficient condition on $h(t)$ or $F(t)$ prior to fast unloading is that they are increasing function of time so that the contact area is a monotonically increasing function of time, thus ensuring the validity of Eqs. (5) and (6).

\section{Oliver-Pharr method}

The most widely used method for estimating the contact depth or area is the procedure proposed by Oliver and Pharr. ${ }^{16,17}$ Based on the results of Sneddon, ${ }^{21}$ Oliver and Pharr developed an expression for $h_{\mathrm{c}}$ at the indenter displacement $h_{\mathrm{m}}$,

$$
h_{\mathrm{c}}=h_{\mathrm{m}}-\xi \frac{F_{\mathrm{m}}}{(\mathrm{d} F / \mathrm{d} h)_{\mathrm{m}}},
$$

where $F_{\mathrm{m}}$ and $(\mathrm{d} F / \mathrm{d} h)_{\mathrm{m}}$ are the respective load and the initial slope of the unloading curve at the indenter displacement depth $h_{\mathrm{m}}$. The numerical value of $\xi$ is (2/ $\pi)(\pi-2)=0.727$ for conical indenter. Although Eq. (13) was derived from solutions to elastic contact problems, it has been used to estimate contact depth for indentation in elastic-plastic solids ${ }^{16,17}$ and viscoelastic solids. ${ }^{22,23}$ In the following, we examine the conditions for using Eq. (10) and the applicability of Eq. (13) by analyzing the complete loading-unloading curves and contact depths obtained from finite element calculations.

\section{NUMERICAL RESULTS}

\section{A. Finite element model}

We consider a frictionless, rigid conical indenter of half angle $\theta=70.3^{\circ}$ degrees indenting an isotropic linear viscoelastic solid. This indenter half angle is chosen since its depth-to-volume relation is the same as that for the Berkovich indenter so that the results are expected to be applicable to Berkovich indentation.

A three-parameter "standard" linear viscoelastic model is used to describe the shear and hydrostatic relaxation modulus (see Fig. 1): 


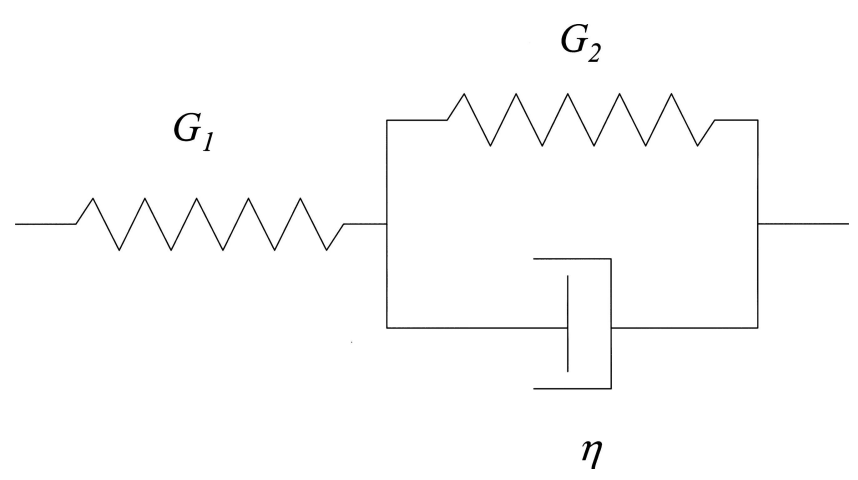

FIG. 1. Three-parameter "standard" model for linear viscoelastic solids.

$$
\begin{aligned}
& G(t)=G_{1}\left[1-\frac{G_{1}}{G_{1}+G_{2}}\left(1-e^{-t / \tau_{\mathrm{s}}}\right)\right] \\
& K(t)=K_{1}\left[1-\frac{K_{1}}{K_{1}+K_{2}}\left(1-e^{-t / \tau_{\mathrm{s}}}\right)\right],
\end{aligned}
$$

where the relaxation time $\tau_{\mathrm{s}}=\eta /\left(G_{1}+G_{2}\right)$. Various parameters are given as $G_{1}=235 \mathrm{MPa}, G_{2}=25.8 \mathrm{MPa}$, $K_{1}=688 \mathrm{MPa}, K_{2}=75.6 \mathrm{MPa}$, and $t=0.99 \mathrm{~s}$. The parameters are chosen such that Poisson's ratio is time independent, though both $G(t)$ and $K(t)$ are time dependent. Specifically, their values at $t=0$ and $t=\infty$ are as follows: $G(0)=235 \mathrm{MPa}$ and $G(\infty)=23.2 \mathrm{MPa}$; $K(0)=688 \mathrm{MPa}$, and $K(\infty)=68.1 \mathrm{MPa}$; and $v=$ 0.483 . The parameters of this fictitious model solid are used for illustrative purposes. Because of linearity, the results can be scaled to represent other materials of the same general type when the dimensionless parameters, such as $G_{1} / G_{2}, K_{1} / K_{2}, G_{1} / K_{1}$, and $t / \tau_{\mathrm{s}}$, are equal. Finite element calculations were carried out using the classical isotropic linear viscoelastic model implemented in ABAQUS (HKS, Inc., Pawtucket, RI) using either displacement or load as the independent variable. The finite element mesh is the same as that used in Ref. 24.

\section{B. Displacement control}

For constant indentation displacement rate profiles given in Fig. 2(a), the corresponding loading-unloading curves from finite element calculations are shown in Fig. 2(b). These examples clearly show that, for the same loading history, the initial unloading slopes converge when unloading rate is sufficiently fast, in agreement with Eq. (9). A tangent line with the converged initial unloading slope is also shown in Fig. 2(b). Furthermore, Fig. 2(b) suggests that the complete unloading curve converges to one limiting case as the unloading rate increases.

\section{Load control}

For constant indentation loading rate profiles given in Fig. 3(a), the corresponding loading-unloading curves


FIG. 2. (a) Displacement-time profiles and (b) calculated loadingunloading curves for the same loading rate and three different unloading rates. The tangent line with initial unloading slope is also shown for the converged unloading curve (b). The loading-unloading curves are labeled by the time duration of unloading.

from finite element calculations are shown in Fig. 3(b). When unloading is slow, the indentation depth continues to increase after the force reaches a maximum, resulting in a "bulge" or "nose" in the unloading curve, which has also been observed experimentally. ${ }^{22-27}$ This bulge is the consequence of the continuing forward movement of the indenter during the early stage of unloading. Because of the forward movement of the indenter, the maximum contact area occurs after the force maximum. This delayed maximum contact area behavior was predicted by the analytical theories of Graham ${ }^{5}$ and Ting, ${ }^{8,9}$ and was verified by numerical calculations. ${ }^{20}$ With increasing unloading rate, the "bulge" disappears. For the same loading history, the initial unloading slope converges when the unloading rate is sufficiently fast, in agreement with Eq. (12). Furthermore, the entire unloading curve converges to one limiting case as the unloading rate increases. 

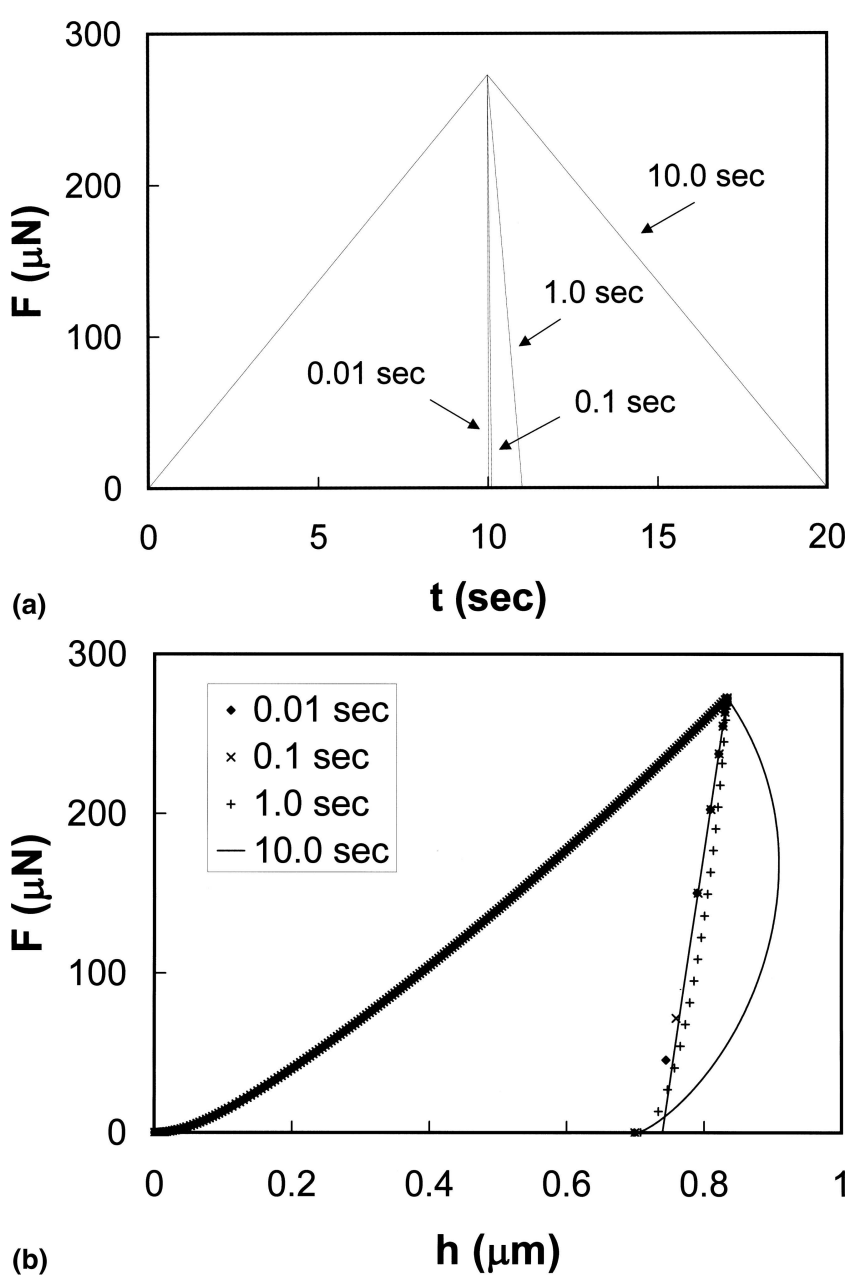

FIG. 3. (a) Force-time profiles and (b) the calculated loadingunloading curves for the same loading rate and four different unloading rates. The tangent line with initial unloading slope is also shown for the converged unloading curve (b). The loading-unloading curves are labeled by the time duration of unloading.

\section{Constant indentation strain rate}

We now consider indentation under the condition of constant indentation strain rates, i.e., $(\mathrm{d} F / \mathrm{d} t) / F$ is a constant, which has been suggested as a preferred method for performing indentation measurements in viscoelastic solids. ${ }^{23}$ We calculate a set of loading-unloading curves with loading-unloading history shown in Fig. 4(a): exponential loading, $F(t)=0.001 \mu \mathrm{N} \cdot \operatorname{EXP}(t / 1.0$ s) [i.e., $\left.(\mathrm{d} F / \mathrm{d} t) / F=1.0 \mathrm{~s}^{-1}\right)$, and linear unloading from the same maximum indentation force with various unloading rates. The corresponding loading-unloading curves shown in Fig. 4(b) demonstrate that the initial unloading slope and the shape of unloading curve are functions of the unloading condition when the loading condition is kept the same. The initial unloading slope and the entire unloading curve converge when unloading rate is sufficiently fast.

The above three examples show that we can define an
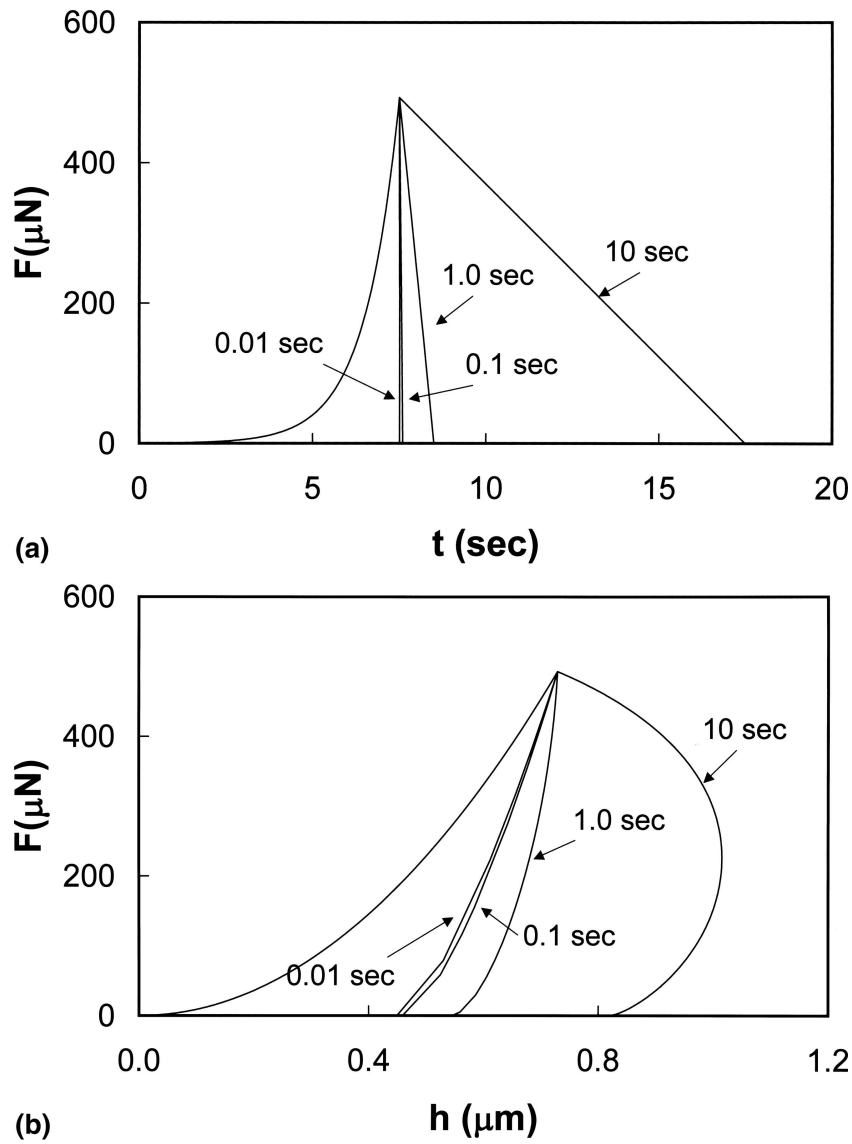

FIG. 4. (a) Exponential loading and linear-unloading from the same maximum indentation load within a time period $0.01,0.1,1.0$, and $10 \mathrm{~s}$ and (b) calculated loading-unloading curves.

unloading curve as the "converged" unloading curve for any given loading history.

\section{E. Contact depth and unloading slope}

The contact area $A$ and contact depth $h_{\mathrm{c}}$ are also obtained from finite element calculations. The calculations show $h_{\mathrm{c}} / h \approx 0.658 \pm 0.001$, which is larger than $2 / \pi \approx$ 0.636 predicted by Eq. (8). This suggests that Eq. (8) needs to be slightly modified to become

$$
\frac{h_{\mathrm{c}}}{h}=\alpha \frac{2}{\pi},
$$

where $\alpha=1.034 \pm 0.002$. Furthermore, the finite element results show that there is a small correction to Eq. (10),

$$
\left.\frac{\mathrm{d} F}{\mathrm{~d} h}\right|_{h=h_{\mathrm{m}}}=\beta \frac{4 G(0) \tan \theta}{1-v} h_{\mathrm{c}}=\beta \frac{2}{\sqrt{\pi}} \frac{E(0)}{1-v^{2}} \sqrt{A},
$$

where $\beta=1.06 \pm 0.02$. The same $\beta$ correction factor has also been seen in the modeling of indentation in purely 
elastic solids and in elastic-plastic solids. ${ }^{17,20}$ The origin of this correction factor has been discussed previously. ${ }^{17,20}$ It has been attributed to the fact that Eq. (10) was derived using linearized boundary conditions and infinitesimal theory of continuum mechanics; finite element calculations take into account nonlinear effects, including large strain and moving contact boundaries. ${ }^{20}$ Because the values of $\alpha$ and $\beta$ are nearly the same as those for purely elastic and elastic-plastic cases, we believe they are insensitive to the particular choice of viscoelastic properties used in the finite element calculations. Although further improvement in the precision and accuracy of the $\alpha$ and $\beta$ values is possible by additional calculations, Eqs. (15) and (16) are expected to be more accurate than the corresponding Eqs. (8) and (10) for determining contact depth $h_{\mathrm{c}}$ and "instantaneous" moduli, $G(0) /(1-v)$ or $E(0) /\left(1-v^{2}\right)$, when unloading is sufficiently fast.

\section{F. Oliver-Pharr method}

We now discuss the applicability of the Oliver-Pharr method for estimating contact depth for indentation in viscoelastic solids. Finite element calculations were carried out using constant indentation displacement rate profiles given in Fig. 5(a). The load-displacement curves in Fig. 5(b) show that the force required to reach a given indentation depth increases with the loading rate, consistent with the expected behavior of viscoelastic solids. The unloading rates chosen in the calculations are sufficiently fast so that they generate the corresponding converged unloading curves. Furthermore, finite element calculations show that the contact depth $h_{\mathrm{c}}$ is the same for all three cases shown in Fig. 5(b), as expected from Eq. (15) since $h_{\mathrm{m}}$ is the same. According to Eq. (16), therefore, the unloading slopes are the same, which is evident from Fig. 5(b).

On the other hand, Fig. 5(b) demonstrates that the Oliver-Pharr procedure for estimating the contact depth using Eq. (13) is not applicable to indentation in viscoelastic solids. This can be seen by the fact that Eq. (13) would have predicted different contact depths $h_{\mathrm{c}}$ since $F_{\mathrm{m}}$ is different while $h_{\mathrm{m}}$ and $(\mathrm{d} F / \mathrm{d} h)_{\mathrm{m}}$ are the same for the three cases, contradicting the fact that $h_{\mathrm{c}}$ is the same. Indeed, the contact depths calculated from the OliverPharr procedure for the three cases shown in Fig. 5(b) are $0.34,0.43$, and $0.48 \mu \mathrm{m}$ for the $0.5,5.0$, and $50 \mathrm{~s}$ loading times, respectively. In contrast, the actual contact depth for all three cases is $0.33 \mu \mathrm{m}$. The errors are $3 \%, 30 \%$, and $45 \%$. Errors of the same magnitude are also seen from finite element calculations when load, instead of displacement, is the independent variable. Thus, the Oliver-Pharr procedure may cause significant error in determining contact depth or contact area when it is applied to the analysis of indentation in viscoelastic solids.


FIG. 5. (a) Displacement-time profiles and (b) calculated loadingunloading curves for three different loading rates and sufficiently fast unloading rates. The tangent lines with initial unloading slopes are also shown in (b). The loading-unloading curves are labeled by the time duration of loading.

\section{CONCLUSIONS}

Using analytical and finite element modeling, we have established a relationship between contact depth and indenter displacement [Eq. (15)] and a relationship between initial unloading slope, contact depth, and the instantaneous modulus for sufficiently high rate of unloading [Eq. (16)]. We have shown that the Oliver-Pharr method for estimating the contact depth [Eq. (13)] is not applicable to indentation in viscoelastic solids.

These analytical and numerical results form the basis for performing indentation measurements in linear viscoelastic solids. Specifically, Eqs. (9) and (12) imply that:

(1) Fast unloading is essential in determining the instantaneous modulus from initial unloading slope using Eq. (16). The numerical examples in this paper suggest 
that "sufficiently fast" unloading is achieved when the time duration of liner unloading is about 0.1 to 0.01 times the relaxation time of linear viscoelastic materials. In practice, the manifestation of sufficiently fast unloading is the "converged" unloading curve that can be obtained using different unloading rates spanning several order of magnitudes. The development and improvement of indentation equipment that allow fast and slow responses are thus encouraged.

(2) A number of authors have suggested adding a "hold-at-peak-load" period between loading and unloading to avoid the occurrence of the "bulge" on the unloading curves. ${ }^{23,25-30}$ It has been shown recently that the initial unloading slope is nevertheless a function of holding history and unloading condition. ${ }^{23,25-30}$ A correction formula has been proposed by Ngan et al. for the initial unloading slope after the hold period so that the instantaneous modulus can be obtained from the unloading slope. ${ }^{27-30}$ Eqs. (9) and (12) suggest, however, that the hold period may be unnecessary if unloading can be made sufficiently fast so that the instantaneous modulus can be obtained from the unloading slope.

(3) There has been much discussion in the literature on the constant indentation strain-rate loading condition, i.e., constant $(\mathrm{d} F / \mathrm{d} t) / F$ or $(\mathrm{d} h / \mathrm{d} t) / h$ for measuring the time-dependent properties of materials using conical or pyramidal indenters. ${ }^{23,31}$ It has been shown both experimentally $^{31}$ and theoretically ${ }^{32}$ that the hardness of power-law creep solids is proportional to $[(\mathrm{d} F / \mathrm{d} t) / F]^{m}$, where $m$ is the creep exponent. Consequently, the hardness value is depth-independent under the constant indentation strain rate loading conditions, whereas it is depth-dependent under other loading conditions such as constant loading rate or constant displacement rate. Furthermore, the creep exponent can be obtained from hardness measurements using several indentation strain rates. Therefore, constant indentation strain rate measurements are particularly useful for measuring the properties of power-law creep solids. In contrast, Eq. (9) and (12) show that loading methods, either in displacement control or load control, are unimportant for measuring the instantaneous modulus of viscoelastic solids, as long as the unloading rate is sufficiently fast. It seems, therefore, there is little advantage of using the constant indentation strain rate method for measuring the instantaneous modulus of viscoelastic solids.

(4) When the effect of tip rounding can be neglected, a simple relationship exists for determining the contact depth from indentation depth [Eq. (15)].

These general guidelines should help improve the accuracy and reproducibility of conical indentation measurements for determining properties of purely linear viscoelastic solids. Although this work focuses on conical indentation, similar conclusions also hold true for spherical indentation in linear viscoelastic solids. ${ }^{33}$

\section{ACKNOWLEDGMENTS}

The authors would like to thank Wangyang Ni, Mike Lukitsch, Yue Qi, Tom Perry, Wes Capehart, Lou Hector, and Mark W. Verbrugge for valuable discussions. C-M. Cheng would like to acknowledge partial support from the National Science Foundation of China, Project No. 10372101.

\section{REFERENCES}

1. E.H. Lee: Stress analysis in visco-elastic bodies. Quarterly Appl. Math. 13, 183 (1955).

2. J.R.M. Radok: Visco-elastic stress analysis. Quarterly Appl. Math. 15, 198 (1957).

3. E.H. Lee and J.R.M. Radok: The contact problem for viscoelastic bodies. J. Appl. Mech. 27, 438 (1960).

4. S.C. Hunter: The Hertz problem for a rigid spherical indenter and a viscoelastic half-space. J. Mech. Phys. Solids 8, 219 (1960).

5. G.A.C. Graham: The contact problem in the linear theory of viscoelasticity. Int. J. Eng. Sci. 3, 27 (1965).

6. G.A.C. Graham: Contact problem in linear theory of viscoelsticity when time dependent contact area has any number of maxima and minima. Int. J. Eng. Sci. 5, 495 (1967).

7. W.H. Yang: Contact problem for viscoelastic bodies. J. Appl. Mech. 33, 395 (1966).

8. T.C.T. Ting: Contact stresses between a rigid indenter and a viscoelastic half-space. J. Appl. Mech. 33, 845 (1966).

9. T.C.T. Ting: Contact problems in linear theory of viscoelasticity. J. Appl. Mech. 35, 248 (1968).

10. L. Cheng, X. Xia, W. Yu, L.E. Scriven, and W.W. Gerberich: Flat-punch indentation of viscoelastic material. J. Polym. Sci. Part B: Polym. Phys. 38, 10 (2000).

11. P-L. Larrson and S. Carlsson: On microindentation of viscoelastic polymers. Polym. Test. 17, 49 (1998).

12. S. Shimizu, T. Yanagimoto, and M. Sakai: Pyramidal indentation load-depth curve of viscoelastic materials. J. Mater. Res. 14, 4075 (1999).

13. M. Sakai and S. Shimizu: Indentation rheometry for glass-forming materials. J. Non-Cryst. Solids 282, 236 (2001).

14. M. Sakai: Time-dependent viscoelastic relation between load and penetration for an axisymmetric indenter. Philos. Mag. A82, 1841 (2002).

15. M.L. Oyen and R.F. Cook: Load-displacement behavior during sharp indentation of viscous-elastic-plastic materials. J. Mater. Res. 18, 139 (2003).

16. W.C. Oliver and G.M. Pharr: An improved technique for determining hardness and elastic modulus using load and displacement sensing indentation experiments. J. Mater. Res. 7, 1564 (1992).

17. W.C. Oliver and G.M. Pharr: Measurement of hardness and elastic modulus by instrumented indentation: Advances in understanding and refinements to methodology. J. Mater. Res. 19, 3 (2004).

18. W.N. Findley, J.S. Lai, and K. Onaran: Creep and Relaxation of Nonlinear Viscoelastic Materials (Dover, NY, 1976).

19. G.T. Mase and G.E. Mase: Continuum Mechanics for Engineers, 2nd Ed. (CRC, Boca Raton, FL, 1999).

20. Y-T. Cheng and C-M. Cheng: Scaling, dimensional analysis, and indentation measurements. Mater. Sci. Eng. R44, 91 (2004).

21. I.N. Sneddon: The relation between load and penetration in the axisymmetric Boussinesq problem for a punch of arbitrary profile. Int. J. Eng. Sci. 3, 47 (1965).

22. M.R. Van Landingham: Review of instrumented indentation. J. Res. Nat. Inst. Stand. Tech. 108, 249 (2003). 
23. B.J. Briscoe, L. Fiori, and E. Pelillo: Nano-indentation of polymeric surfaces. J. Phys. D: Appl. Phys. 31, 2395 (1998).

24. W. Ni, Y-T. Cheng, C-M. Cheng, and D.S. Grummon: An energybased method for analyzing instrumented spherical indentation experiments. J. Mater. Res. 19, 149 (2004).

25. G. Hochstetter, A. Jimenez, and J.L. Loubet: Strain-rate effects on hardness of glassy polymers in the nanoscale range. Comparison between quasi-static and continuous stiffness measurements. J. Macromol. Sci. Phys. B38, 681 (1999).

26. T. Chudoba and F. Richter: Investigation of creep behaviour under load during indentation experiments and its influence on hardness and modulus results. Surf. Coat. Tech. 148, 191 (2001).

27. G. Feng and A.H.W. Ngan: Effects of creep and thermal drift on modulus measurement using depth-sensing indentation. J. Mater. Res. 17, 660 (2002).

28. A.H.W. Ngan and B. Tang: Viscoelastic effects during unloading in depth-sensing indentation. J. Mater. Res. 17, 2604 (2002).
29. B. Tang and A.H.W. Ngan: Accurate measurement of tip-sample contact size during nanoindentation of viscoelastic materials. J. Mater. Res. 18, 1141 (2003).

30. A.H.W. Ngan, H.T. Wang, B. Tang, and K.Y. Sze: Correcting power-law viscoelastic effects in elastic modulus measurement using depth-sensing indentation. Int. J. Solids Struct. 42, 1831 (2005).

31. B.N. Lucas and W.C. Oliver: Indentation power-law creep of high-purity indium. Metall. Mater. Trans. A 30, 601 (1999).

32. Y-T. Cheng and C-M. Cheng: Scaling relationships in indentation of power-law creep solids using self-similar indenters. Philos. Mag. Lett. 81, 9 (2001).

33. Y-T. Cheng and C-M. Cheng: Relationships between initial unloading slope, contact depth, and mechanical properties for spherical indentation in linear viscoelastic solids. GM R\&D Publication $R \& D .10,059$ (Feb. 21, 2005). 